\title{
Association between BDNF G196A (Val66Met) polymorphism and cognitive impairment in patients with Parkinson's disease: a meta-analysis
}

\author{
Qian Wang (ib), Jianfang Liu (i), Yikun Guo (ib), Guanzhong Dong (i), Wenying Zou (iD), and \\ Zhuoyou Chen (iD \\ Department of Neurology, the Affiliated Changzhou No. 2 People's Hospital of Nanjing Medical University, \\ Changzhou, Jiangsu, China
}

\begin{abstract}
Brain-derived neurotrophic factor (BDNF) is widely expressed in the central nervous system and prolongs the survival of dopaminergic neurons in the substantia nigra. Several studies have recently investigated the association between BDNF G196A (Val66Met), a single nucleotide polymorphism influencing cognitive processes, and cognitive impairment in Parkinson's disease (PD), but with contradictory findings. Thus, this meta-analysis was performed to clarify the possible association. Relevant studies were identified by a systematic search of PubMed, Embase, and China National Knowledge Infrastructure (CNKI) databases. The strength of the association was evaluated using crude odds ratios and $95 \%$ confidence interval. Finally, six studies involving 532 cases and 802 controls were included. Our analyses suggested the G196A (Val66Met) polymorphism was significantly associated with cognitive impairment in PD, especially in Caucasian populations. In conclusion, BDNF G196A (Val66Met) is confirmed to be a risk factor for cognitive impairment in PD.
\end{abstract}

Key words: BDNF; Meta-analysis; Cognitive impairment; Parkinson's disease

\section{Introduction}

Parkinson's disease (PD) is a neurodegenerative disease characterized by the degeneration and loss of dopaminergic neurons in the substantia nigra and the formation of Lewy bodies. The main clinical manifestations of PD are tremor, myotonia, bradykinesia, and abnormal posture and gait. Non-motor symptoms (e.g. olfactory dysfunction, cognitive impairment, sleep disorder, pain, depression, anxiety, autonomic nervous dysfunction) are other important clinical manifestations running throughout all stages of PD and occur earlier than motor symptoms, seriously reducing the quality-of-life (1). As one of the most severe and disabling non-motor symptoms of PD, cognitive impairment is closely related to the mortality of the disease and thus has attracted wide attention. Cognitive impairment not only seriously impairs social function and the quality-of-life of PD patients, but also intensifies the burden of caregivers and the costs of disease-related medical care, and extends the length of stay (2). Currently, cognitive impairment in PD patients can be divided into two stages: PD with mild cognitive impairment (PDMCl) and PD with dementia (PDD). Epidemiological data show that the prevalence of PDD is
$30 \%$, accounting for $3-4 \%$ of the dementia patients (3). About $10 \%$ of PD patients develop dementia every year, which is 4-6 times higher than that of non-PD patients (4). PDMCl affects $15-20 \%$ of PD patients at early stages; PDD occurs in about $80 \%$ of PD patients during the course of the disease (5).

The pathogenesis of PD is still unclear, but is believed to be caused jointly by genetic and environmental factors and to be related to many mechanisms, such as altered protein handling, reactive microgliosis, oxidative stress, and mitochondrial dysfunction (6). The basic pathological changes of $\mathrm{PD}$ are the chronic progressive degeneration of dopamine neurons in the substantia nigra and the acidification of the microenvironment around dopamine neurons. PD patients were found with neuronal apoptosis and massive proliferation of glial cells (7).

The exact pathogenesis of cognitive impairment of PD is unclear. Neuropathological findings showed that cognitive impairment of PD was correlated with the number of cortical Lewy bodies, Alzheimer's disease (AD)-related pathological changes (such as neurofibrillary tangles, senile plaques), and cerebrovascular lesions. At present, 
the specific susceptibility genes for cognitive impairment of PD are unknown. The brain-derived neurotrophic factor (BDNF), which can significantly enhance the tolerance of dopamine neurons against the acidic environment, is significantly downregulated in the ventral substantia nigra of PD patients (8). G196A (Val66Met, rs6265) polymorphism, with a valine (Val) to methionine (Met) substitution at codon 66 , is one of the most frequently studied BDNF gene polymorphisms.

Several recent studies described the association between BDNF G196A (Val66Met, rs6265) polymorphism and cognitive impairment in PD, but reported conflicting and inconclusive findings (9-14). There may be several reasons for the different conclusions. First, diagnostic criteria for cognitive impairment in patients with PD differs greatly. Due to the lack of unified diagnostic criteria, some studies adopt relatively loose criteria, while others adopt more stringent diagnostic criteria, which leads to great differences in research conclusions. Second, dyskinesia such as static tremor, bradykinesia, rigidity, and non-motor symptoms such as pain and fatigue in PD patients may interfere with the accuracy and reliability of cognitive function assessment. Finally, the different age composition and sample size of the subjects will also lead to different research results. In an attempt to resolve these contradictory findings, we performed this meta-analysis involving 6 studies.

\section{Material and Methods}

\section{Inclusion criteria}

Inclusion criteria were: 1) case-control studies investigating the association between gene BDNF G196A (Val66Met) polymorphism and cognitive impairment in PD; 2) sufficient data for calculating the pooled odds ratio (ORs) with 95\% confidence interval (Cl); 3) studies on humans. Exclusion criteria were 1) duplication; 2) case report or review; 3) article with other genetics polymorphisms and in vitro experiments.

\section{Search strategy}

Two investigators independently and systematically searched PubMed, EMBASE, and China National Knowledge Infrastructure (CNKI) databases online up to October 1, 2018 to identify relevant studies using the following keywords: ("BDNF G196A" or "BDNF Val66Met" or "rs6265") AND ("genetic polymorphism" or "single nucleotide polymorphisms" or "SNP") AND ("cognitive impairment" or "dementia") AND ("Parkinson's disease" or "PD"). References in the cited studies and reviews were also checked for other relevant publications. When two studies overlapped, we chose the latest study or the one with a larger sample size. There was no restriction on language, ethnicity, or region of study population. Cognitive impairment was diagnosed according to classification criteria.

\section{Data extraction and quality assessment}

Two investigators reviewed and extracted data independently in accordance with the inclusion criteria. The following information from each study was collected: surname of first author, year of publication, country, ethnicity, sample size, and genotype distributions among cases and controls. When a study involved more than one ethnicity, the genotype data were extracted separately. The quality of the selected studies was assessed using the Newcastle-Ottawa Scale (NOS). Analyses were based on three broad dimensions of selection, comparability, and exposure in the case-control studies. The NOS scores vary from 0 up to 9 : high quality $(>7)$; medium quality $(4-6)$; poor quality $(<4)$. Only studies with more than 5 NOS stars were included. The discrepancies were resolved by discussion or consulting with a third reviewer.

\section{Statistical analysis}

Pooled ORs with $95 \% \mathrm{Cl}$ were calculated to evaluate the strength of relationship between BDNF gene rs6265 polymorphism and cognitive impairment in PD. Subgroup analyses were performed according to ethnicity. Heterogeneity between studies was detected by the chi squaredbased $Q$-test. $\mathrm{P}<0.10$ or $\mathrm{I}^{2}>50 \%$ was considered to be significant heterogeneity and a random-effects model was applied; otherwise a fixed-effect model was used. Sensitivity analysis was conducted by excluding studies one-by-one to evaluate the effect of individual studies on the pooled ORs and evaluate the stability of the results. A $\chi^{2}$ test was used to determine whether the observed genotype frequencies conformed to the Hardy-Weinberg equilibrium (HWE). Publication bias was evaluated by visual inspection of symmetry of Begg's funnel plot and by Egger's test (statistical significance at $P<0.05$ ). All statistical analyses were performed on STATA 11.0.

\section{Results}

\section{Characteristics of the included publications}

The initial search returned a total of 20 articles. Figure 1 represents a flow chart of the retrieved and excluded studies with the reasons for exclusion. Fourteen articles were excluded, including 2 reviews, 9 irrelevant articles, and 3 articles without reporting detailed genotype data. Finally, 6 publications were enrolled, consisting of 532 cases and 802 controls. The characteristics of the selected studies are presented in Table 1. Genotype distributions of rs6265 of the controls conformed to HWE $(P>0.05)$. The NOS scores of the included studies ranged from 5 to 7 stars, suggesting they were of high methodological quality. Five papers were carried out in Caucasian populations and 1 in Asian population.

\section{Quantitative analysis}

Allele and genotype distributions of the BDNF G196A polymorphism from each included study are shown in 
Table 2. rs6265 polymorphism was significantly associated with cognitive impairment in PD in 2 models (AA vs $G A+G G$ and $A A$ vs GA; Figure 2). Stratification analyses showed this association was significant among Caucasian populations in 3 models (AA vs $G A+G G, A A$ vs $G G$, and AA vs GA; Figure 3).

\section{Sensitivity analysis and publication bias}

Sensitivity analysis showed that the omission of any individual study did not substantially influence the risk estimates, which supported the credibility and reliability of this meta-analysis.

Begg's funnel plot or quantitative Egger's test did not find any obvious publication bias for the association between BDNF G196A (Val66Met) and cognitive impairment in PD.

\section{Discussion}

PDMCI is often considered an early manifestation of cognitive decline in PD and a transitional state for the subsequent progression to PDD (15). PDMCl is defined as having at least one cognitive domain (memory, visual

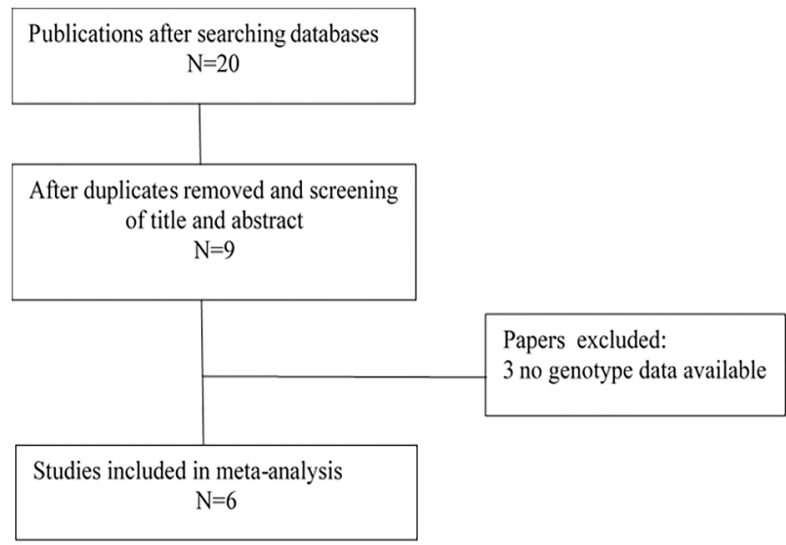

Figure 1. Selection of publications included in this meta-analysis. space, execution) below 1.5 standard deviations and not meeting the dementia criteria compared with the same age group (16). The pathological study by Braak et al. on PD showed that the lesions of Lewy bodies increased with the prolonged onset time from olfactory bulb and preolfactory nucleus to limbic system and neocortex (17). It can be concluded that more brain regions related to cognitive function are damaged as the disease course is lengthened, which, however, cannot explain the cognitive dysfunction in early stage of PD. Moreover, Braak's pathological stage reflects only the deposition of Lewis bodies but not the neuron damage or synaptic dysfunction. AD-related pathological changes (neurofibrillary tangles, senile plaques) play an equally or even more important role than the number of cortical Lewy bodies in the neuropathological changes of cognitive decline of PD (18). In addition, cerebrovascular lesions were confirmed in some PD patients with cognitive dysfunction (19). Imaging and clinical studies showed two possible subtypes of cognitive impairment in PD patients: fronto-striatal deficits (attention, memory, and speech fluency abnormalities featured by relatively slow progression), and posterior-cortical deficits (visual anecdote and memory abnormalities, such as naming and retelling) (20). While fronto-striatal defects are more related to dopaminergic defects and reactive to dopaminergic manipulation, the degeneration of cholinergic projection fibers from the basal forebrain is a highly probable correlate of posterior-cortical defects and is pivotal in PDD development. In addition to dopamine, neurotransmitters such as serotonin, norepinephrine, and choline are also associated with cognitive impairment. Executive function, free recall, and visual spatial ability are associated with abnormal cholinergic function, which may be one of the causes for cognitive impairment in PD.

Because of the family clustering of PDD, it is speculated that there may be some genetic susceptibility to cognitive impairment of PD (21). Although great progress has been made in the study of pathogenic and susceptible genes of $P D$, the genes related to cognitive impairment of PD have not been fully identified. There are only a few widely recognized susceptibility genes, such as

Table 1. Characteristics of included studies.

\begin{tabular}{|c|c|c|c|c|c|c|c|c|c|c|}
\hline \multirow[t]{2}{*}{ Author, year (ref n.) } & \multirow[t]{2}{*}{ Country } & \multirow[t]{2}{*}{ Ethnicity } & \multicolumn{3}{|c|}{ Case } & \multicolumn{3}{|c|}{ Control } & \multirow[t]{2}{*}{ HWE } & \multirow[t]{2}{*}{ NOS } \\
\hline & & & GG & GA & AA & GG & GA & $\mathrm{AA}$ & & \\
\hline Altmann, 2016 (11) & Brazil & Caucasian & 56 & 33 & 5 & 61 & 18 & 2 & 0.632 & 6 \\
\hline Bialecka, 2014 (10) & Poland & Caucasian & 143 & 47 & 5 & 33 & 15 & 1 & 0.636 & 6 \\
\hline Wang, 2014 (12) & China & Asian & 54 & 81 & 39 & 22 & 49 & 18 & 0.330 & 7 \\
\hline Karakasis, 2011 (13) & Greece & Caucasian & 10 & 7 & 1 & 105 & 55 & 6 & 0.714 & 5 \\
\hline Gao, 2010 (14) & Spain & Caucasian & 14 & 6 & 2 & 85 & 63 & 4 & 0.052 & 6 \\
\hline Guerini, 2009 (9) & Italy & Caucasian & 12 & 10 & 7 & 145 & 106 & 14 & 0.339 & 7 \\
\hline
\end{tabular}

HWE: Hardy-Weinberg equilibrium; NOS: Newcastle-Ottawa Scale. 
Table 2. Meta-analysis of associations between the Val66Met polymorphism and cognitive impairment in patients with Parkinson's disease.

\begin{tabular}{|c|c|c|c|c|c|c|c|}
\hline Comparison & $\begin{array}{c}\text { Overall and } \\
\text { Stratification analyses }\end{array}$ & Studies & OR $(95 \% \mathrm{Cl})$ & $\mathrm{P}$ value & $\begin{array}{l}\text { Random/Fixed } \\
\text { effect model }\end{array}$ & $\begin{array}{c}\mathrm{P} \text { for } \\
\text { heterogeneity }\end{array}$ & $\mathrm{I}^{2}(\%)$ \\
\hline \multirow[t]{3}{*}{$A v s \mathrm{G}$} & Overall & 6 & $1.32(0.85,2.07)$ & 0.216 & Random & 0.002 & 72.9 \\
\hline & Caucasian & 5 & $1.46(0.88,2.42)$ & 0.138 & Random & 0.013 & 68.5 \\
\hline & Asian & 1 & $0.90(0.63,1.29)$ & 0.564 & & & \\
\hline \multirow[t]{3}{*}{$A A$ vs $G A+G G$} & Overall & 6 & $1.70(1.06,2.75)$ & 0.029 & Fixed & 0.154 & 37.8 \\
\hline & Caucasian & 5 & $3.06(1.47,6.34)$ & 0.003 & Fixed & 0.616 & 0 \\
\hline & Asian & 1 & $1.14(0.61,2.14)$ & 0.684 & & & \\
\hline \multirow[t]{3}{*}{$A A+G A$ vs $G G$} & Overall & 6 & $1.09(0.82,1.46)$ & 0.548 & Fixed & 0.109 & 44.4 \\
\hline & Caucasian & 5 & $1.26(0.90,1.78)$ & 0.179 & Fixed & 0.166 & 38.3 \\
\hline & Asian & 1 & $0.73(0.41,1.30)$ & 0.286 & & & \\
\hline \multirow[t]{3}{*}{ AA vs GG } & Overall & 6 & $1.64(0.97,2.76)$ & 0.062 & Fixed & 0.104 & 45.3 \\
\hline & Caucasian & 5 & $3.16(1.49,6.67)$ & 0.003 & Fixed & 0.643 & 0 \\
\hline & Asian & 1 & $0.88(0.42,1.86)$ & 0.743 & & & \\
\hline \multirow[t]{3}{*}{ AA vs GA } & Overall & 6 & $1.79(1.09,2.96)$ & 0.022 & Fixed & 0.315 & 15.4 \\
\hline & Caucasian & 5 & $2.79(1.30,6.01)$ & 0.009 & Fixed & 0.554 & 0 \\
\hline & Asian & 1 & $1.31(0.68,2.54)$ & 0.423 & & & \\
\hline
\end{tabular}

Data in bold are significant.

microtubule-associated protein tau (MAPT) gene (22), glucocerebrosidase (GBA) gene23, catechol-O-methyltransferase (COMT) gene (24), dual-specificity tyrosine phosphorylation-regulated kinase $1 \mathrm{~A}$ (DYRK1A) gene (25), and BDNF gene.

BDNF isolated from pig brain plays an important role in promoting the proliferation, regeneration, synaptic remodeling, and regulated release of neurotransmitters (26). The human BDNF coding gene is located in the short arm p13 of chromosome 11 and consists of 11 exons, which can encode a precursor peptide that is protease-hydrolyzed into mature BDNF protein. The amino acid coding sequence of human BDNF is strikingly similar to that of nerve growth factor (NGF), so BDNF is classified as a member of the NGF family (27). BDNF, which is widely distributed in cortical and subcortical areas, maintains dopaminergic neuronal survival and promotes synaptic plasticity, dendritic morphogenesis, arborization, and even neurogenesis in the adult brain. BDNF is crucial for proper establishment of dopaminergic neurons in the substantia nigra of the developing brain (28). Because BDNF gene participates in many functions of the nervous system, it has been widely studied in cognitive dysfunction and psychobehavioral disorders. G196A (Val66Met) is a frequent single nucleotide polymorphism in the targeting region of the human BDNF and is associated with abnormal intracellular trafficking and the BDNF regulated secretion in the cultured hippocampal neurons transfected with the Met allele. The Met allele is associated with hippocampal neuronal dysfunction and impaired episodic memory in humans (29). The Met allele seems to decrease neuronal dendrites distribution and decrease targeting to secretory granules, lowering the extracellular BDNF levels (30). PD patients that were BDNF Met carriers suffered cognitive impairment more frequently and therefore had lower MiniMental State Examination scores than Val/Val homozygotes $(9,11)$. PD patients with BDNF Met alleles, especially women taking dopamine drugs, completed the Tower of London tests better than those with BDNF Val alleles, indicating that BDNF might act on dopamine and its receptors in the limbic system and thereby affect the cognitive function of these patients (31). However, contradictory conclusions were also reported to support a null association $(10,12,13,32,33)$. These discrepancies might be due to different methodologies to assess cognitive impairment. Different cut-offs might have been considered as cognitive impairment in different studies due to differences in years of schooling.

A meta-analysis in 2015 found no association between Val66Met polymorphism and the risk of developing PD in the overall analysis, or between the BDNF G196A genotype and cognitive impairment in the clinical data summarization, but no subgroup analysis of races was performed (34). Lee et al. (35) considered 12 studies in the case of the BDNF $196 \mathrm{G} / \mathrm{A}$ polymorphism, and the meta-analysis showed no association between PD and the BDNF 196A allele in all study subjects, however, ethnicity-specific meta-analysis identified an association between the BDNF $196 \mathrm{AA}+\mathrm{AG}$ genotype and PD in Europeans, but not in Asians. Zintzaras and Hadjigeorgiou (36) and Dai et al. (37) also found no association between BDNF 196 G/A polymorphism and PD. None of the three studies analyzed the relationship between BDNF and cognitive impairment in PD patients. 


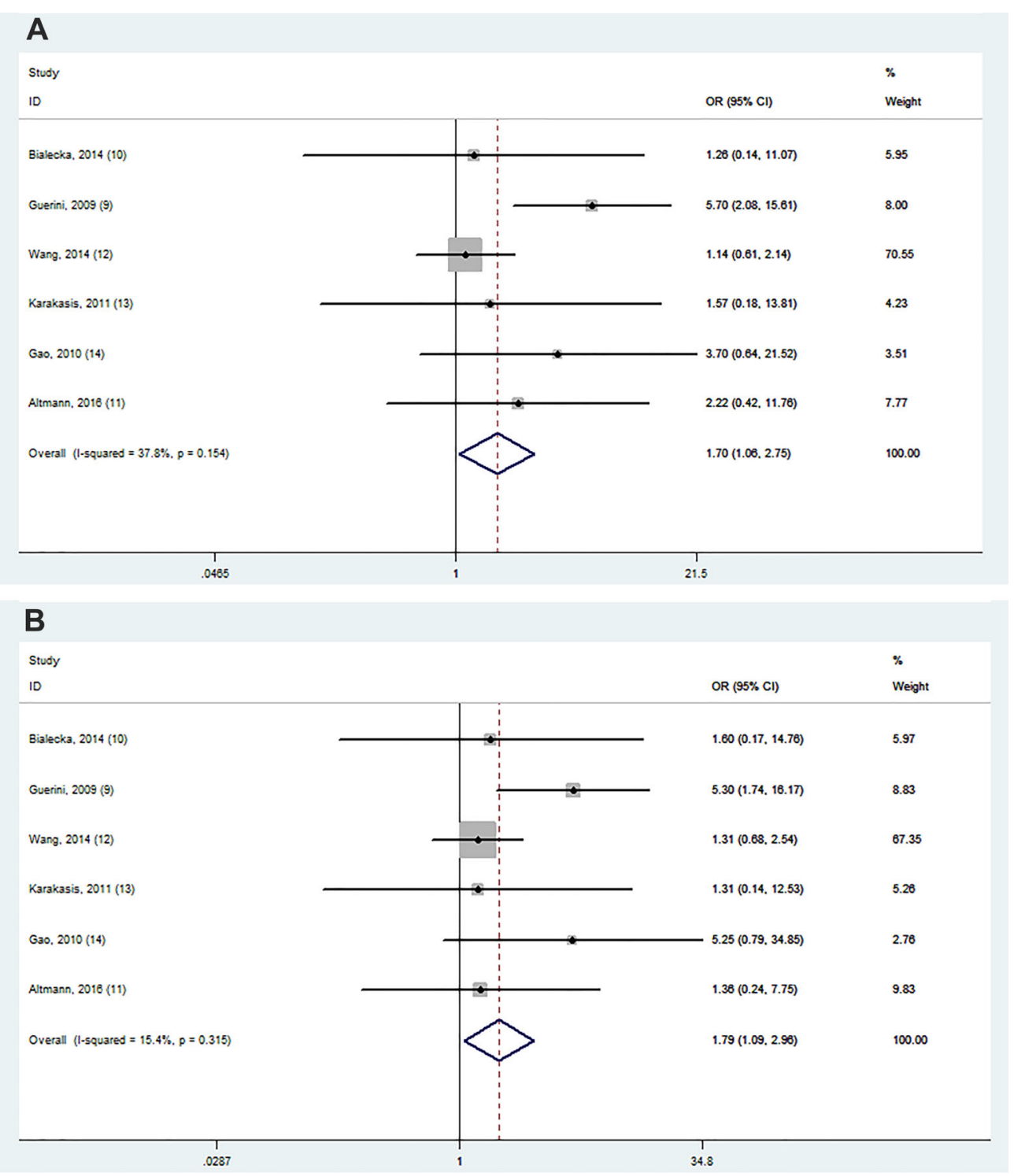

Figure 2. Forest plot showing odds ratio for the associations between rs6265 and cognitive impairment in PD (A: AA vs GA + GG; B: AA vs GA).

We performed this meta-analysis to resolve the contradictory results of all relevant studies published up to 2018. Our analyses suggested that the G196A (Val66Met) polymorphism was significantly associated with cognitive impairment in PD, especially in Caucasian populations. The only study involving the Asian population (12) showed no significant difference in allele or genotype frequencies among non-PD, PDMCI, and PDD groups.

Several potential limitations of this meta-analysis should be considered. First, no subgroup analysis of gender, age of onset, educational level, duration of illness, or genotype methods was conducted owing to the limited number of articles and the small sample size. Second, this meta-analysis only included Asian and Caucasian populations, which calls for future studies on other ethnic groups because of ethnic differences in gene polymorphisms. Third, there was no uniform diagnosis of Parkinson's cognitive impairment in these studies. In 2007, the Movement Disorder Society Task Force (MDS-TF) issued a unified and standardized PDD diagnostic guide and revised it, but the four core cognitive domains were placed in the same weight, and any two cognitive domains with significant impairment could be diagnosed as PDD (38). However, there was also no unified diagnostic criterion for PDMCI. Some studies adopted loose diagnostic criteria, while others used stringent criteria, which led to large 


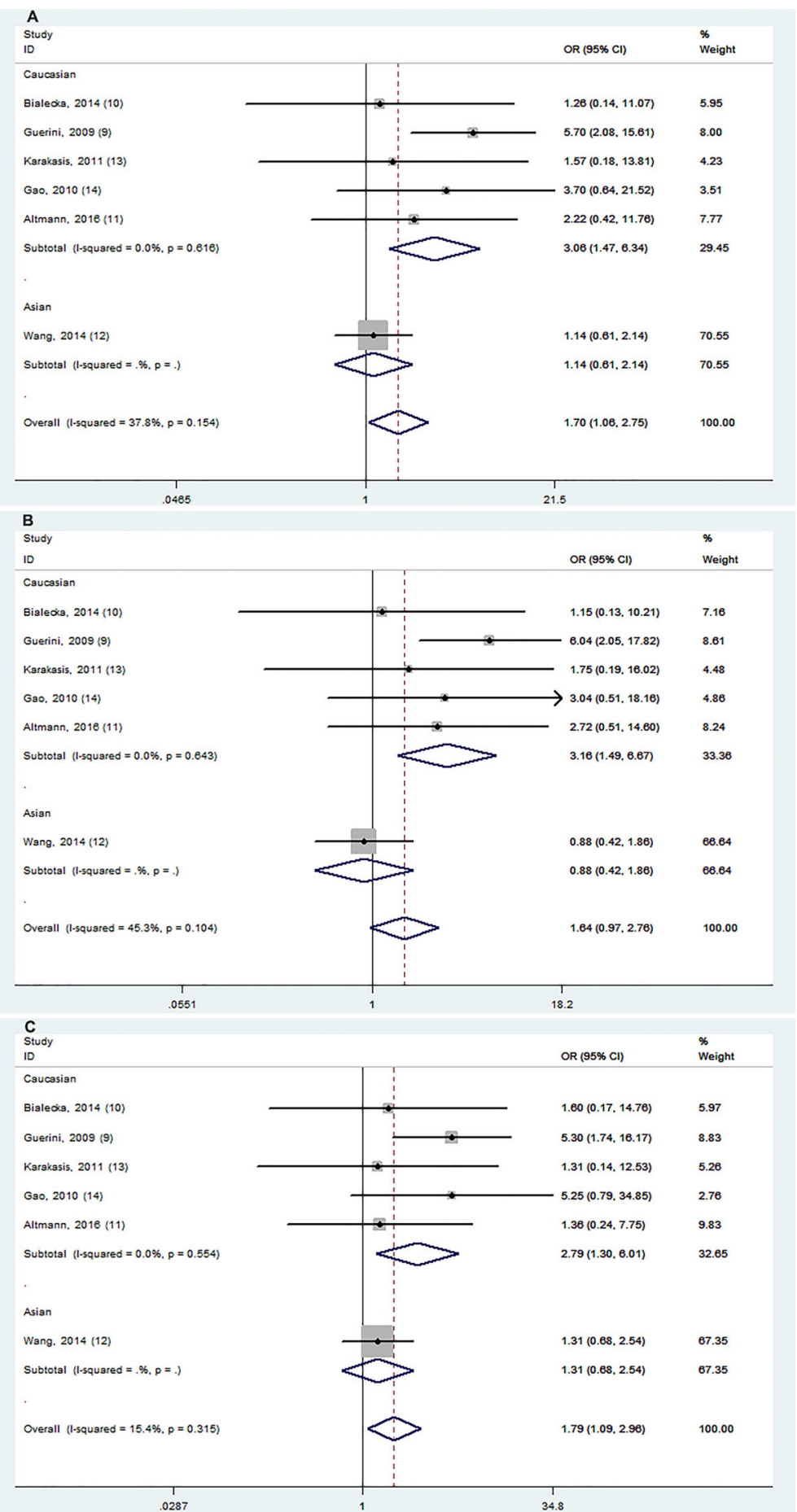

Figure 3. Stratification analyses of ethnicity between rs6265 and cognitive impairment in PD (A: AA vs GA + GG; B: AA vs GG; C: AA vs $\mathrm{GA})$. 
differences in the research conclusions. In 2012, MDS-TF issued a new diagnostic guideline for $\mathrm{PDMCl}$, which corresponded to the characteristics of early cognitive impairment of PD and was well consistent with previous PDD diagnostic guidelines (39).

In conclusion, the BDNF gene rs6265 polymorphism plays important roles in cognitive impairment in Parkinson's disease, especially among Caucasian populations.

\section{References}

1. Postuma RB, Berg D, Stern M, Poewe W, Olanow CW, Oertel W, et al. MDS clinical diagnostic criteria for Parkinson's disease. Mov Disord 2015; 30: 1591-1601, doi: $10.1002 / \mathrm{mds} .26424$

2. Winter $\mathrm{Y}$, von Campenhausen $\mathrm{S}$, Arend M, Longo K, Boetzel $\mathrm{K}$, Eggert $\mathrm{K}$, et al. Health-related quality of life and its determinants in Parkinson's disease: results of an Italian cohort study. Parkinsonism Relat Disord 2011; 17: 265-269, doi: 10.1016/j.parkreldis.2011.01.003

3. Aarsland D, Zaccai J, Brayne C. A systematic review of prevalence studies of dementia in parkinson's disease. Mov Disord 2005; 20: 1255-1263, doi: 10.1002/mds.20527.

4. Aarsland D, Andersen K, Larsen JP, Lolk A, Nielsen H, Kragh-Sorensen P. Risk of dementia in Parkinson's disease: a community-based, prospective study. Neurology 2001; 56: 730-736, doi: 10.1212/WNL.56.6.730.

5. O'Callaghan C, Lewis SJG. Cognition in Parkinson's disease. Int Rev Neurobiol 2017; 133: 557-583, doi: 10.1016/ bs.im.2017.05.002.

6. Yun JW, Ahn JB, Kang BC. Modeling Parkinson's disease in the common marmoset (Callithrix jacchus): overview of models, methods, and animal care. Lab Anim Res 2015; 31: 155-165, doi: 10.5625/lar.2015.31.4.155.

7. de Baat C, van Stiphout MAE, Lobbezoo F, van Dijk KD, Berendse HW. Parkinson's disease: pathogenesis, aetiology, symptoms, diagnostics, and its course. Ned Tijdschr Tandheelkd 2018; 125: 509-515, doi: 10.5177/ntvt.2018.10. 18176.

8. Rahmani F, Saghazadeh A, Rahmani M, Teixeira AL, Rezaei $\mathrm{N}$, Aghamollaei $\mathrm{V}$, et al. Plasma levels of brain-derived neurotrophic factor in patients with Parkinson disease: a systematic review and meta-analysis. Brain Res 2019; 1704: 127-136, doi: 10.1016/j.brainres.2018.10.006.

9. Guerini FR, Beghi E, Riboldazzi G, Zangaglia R, Pianezzola $\mathrm{C}$, Bono $\mathrm{G}$, et al. BDNF val66met polymorphism is associated with cognitive impairment in Italian patients with Parkinson's disease. Eur J Neurol 2009; 16: 1240-1245, doi: 10.1111/j.1468-1331.2009.02706.x.

10. Bialecka M, Kurzawski M, Roszmann A, Robowski P, Sitek EJ, Honczarenko K, et al. BDNF G196A (Val66Met) polymorphism associated with cognitive impairment in parkinson's disease. Neurosci Lett 2014; 561: 86-90, doi: 10.1016/ j.neulet.2013.12.051.

11. Altmann V, Schumacher-Schuh AF, Rieck M, CallegariJacques SM, Rieder CR, Hutz MH. Val66Met BDNF polymorphism is associated with Parkinson's disease cognitive impairment. Neurosci Lett 2016; 615: 88-91, doi: 10.1016/j. neulet.2016.01.030.
Although cognition cannot be considered a pre-motor biomarker of PD, it acts as a staging and prognostic biomarker and plays a key role in sub-typing and disease stratification. Nevertheless, more large-sample studies following the unified diagnostic criteria for cognitive impairment in $\mathrm{PD}$ are needed to further confirm the relationship between BDNF rs6265 and the pathogenesis of PD.

12. Wang Yaqin. Cognitive impairment and genetic susceptibility in Parkinson's disease [Doctor's theis]. Xiangya Hospital, 2014.

13. Karakasis C, Kalinderi K, Katsarou Z, Fidani L, Bostantjopoulou S. Association of brain-derived neurotrophic factor (BDNF) Val66Met polymorphism with Parkinson's disease in a Greek population. J Clin Neurosci 2011; 18: 1744-1745, doi: 10.1016/j.jocn.2011.03.015.

14. Gao L, Diaz-Corrales FJ, Carrillo F, Diaz-Martin J, CaceresRedondo MT, Carballo M, et al. Brain-derived neurotrophic factor g196a polymorphism and clinical features in Parkinson's disease. Acta Neurol Scand 2010; 122: 41-45, doi: 10.1111/j.1600-0404.2009.01253.x.

15. Toribio-Diaz ME, Carod-Artal FJ. [Subtypes of mild cognitive impairment in Parkinson's disease and factors predicting its becoming dementia]. Rev Neurol 2015; 61: 14-24.

16. Aarsland D, Bronnick K, Larsen JP, Tysnes OB, Alves G. Cognitive impairment in incident, untreated Parkinson disease: the Norwegian Parkwest Study. Neurology 2009; 72: 1121-1126, doi: 10.1212/01.wnl.0000338632.00552.cb.

17. Braak H, de Vos RA, Bohl J, Del Tredici K. Gastric alphasynuclein immunoreactive inclusions in Meissner's and Auerbach's plexuses in cases staged for Parkinson's disease-related brain pathology. Neurosci Lett 2006; 396: 67-72, doi: 10.1016/j.neulet.2005.11.012.

18. Sabbagh MN, Adler CH, Lahti TJ, Connor DJ, Vedders L, Peterson LK, et al. Parkinson disease with dementia: comparing patients with and without Alzheimer pathology. Alzheimer Dis Assoc Disord 2009; 23: 295-297, doi: 10.1097/WAD.0b013e31819c5ef4.

19. Jellinger KA. Mild cognitive impairment in Parkinson disease: heterogenous mechanisms. J Neural Transm 2013; 120: 157-167, doi: 10.1007/s00702-012-0771-5.

20. Pagonabarraga J, Kulisevsky J. Cognitive impairment and dementia in Parkinson's disease. Neurobiol Dis 2012; 46: 590-596, doi: 10.1016/j.nbd.2012.03.029.

21. Kurz MW, Schlitter AM, Larsen JP, Ballard C, Aarsland D. Familial occurrence of dementia and parkinsonism: a systematic review. Dement Geriatr Cogn Disord 2006; 22: 288-295, doi: 10.1159/000095159.

22. Di Maria E, Cammarata S, Parodi MI, Borghi R, Benussi L, Galli M, et al. The h1 haplotype of the tau gene (MAPT) is associated with mild cognitive impairment. J Alzheimers Dis 2010; 19: 909-914, doi: 10.3233/JAD-2010-1285.

23. Sidransky E, Nalls MA, Aasly JO, Aharon-Peretz J, Annesi $\mathrm{G}$, Barbosa ER, et al. Multicenter analysis of glucocerebrosidase mutations in Parkinson's disease. $N$ Engl $\mathrm{J}$ Med 2009; 361: 1651-1661, doi: 10.1056/NEJMoa0901281. 
24. Morley JF, Xie SX, Hurtig HI, Stern MB, Colcher A, Horn S, et al. Genetic influences on cognitive decline in Parkinson's disease. Mov Disord 2012; 27: 512-518, doi: 10.1002/ mds.24946.

25. Jones EL, Aarsland D, Londos E, Ballard C. A pilot study examining associations between dyrk1a and alpha-synuclein dementias. Neurodegener Dis 2012; 10: 229-231, doi: 10.1159/000334759.

26. Seidah NG, Benjannet S, Pareek S, Chrétien M, Murphy RA. Cellular processing of the neurotrophin precursors of NT3 and BDNF by the mammalian proprotein convertases. FEBS Lett 1996; 379: 247-250, doi: 10.1016/0014-5793(95) 01520-5

27. Hohn A, Leibrock J, Bailey K, Barde YA. Identification and characterization of a novel member of the nerve growth factor/brain-derived neurotrophic factor family. Nature 1990; 344: 339-341, doi: 10.1038/344339a0.

28. Harward SC, Hedrick NG, Hall CE, Parra-Bueno P, Milner TA, Pan E, et al. Autocrine BDNF-TrkB signalling within a single dendritic spine. Nature 2016; 538: 99-103, doi: 10.1038/nature19766.

29. Hariri AR, Goldberg TE, Mattay VS, Kolachana BS, Callicott $\mathrm{JH}$, Egan MF, et al. Brain-derived neurotrophic factor val66met polymorphism affects human memory-related hippocampal activity and predicts memory performance. J Neurosci 2003, 23: 6690-6694, doi: 10.1523/JNEUROSCI.23-17-06690.2003.

30. Teh CA, Lee TS, Kuchibhatla M, Ashley-Koch A, Macfall J, Krishnan R, et al. Bipolar disorder, brain-derived neurotrophic factor (BDNF) Val66Met polymorphism and brain morphology. PloS One 2012; 7: e38469, doi: 10.1371/ journal.pone.0038469.

31. Foltynie T, Lewis SG, Goldberg TE, Blackwell AD, Kolachana BS, Weinberger DR, et al. The BDNF Val66Met polymorphism has a gender specific influence on planning ability in Parkinson's disease. J Neurol 2005; 252: 833-838, doi: 10.1007/s00415-005-0756-5.
32. Svetel M, Pekmezovic T, Markovic V, Novakovic I, Dobricic $V$, Djuric $G$, et al. No association between brain-derived neurotrophic factor G196A polymorphism and clinical features of Parkinson's disease. Eur Neurol 2013; 70: 257-262, doi: 10.1159/000352033.

33. van der Kolk NM, Speelman AD, van Nimwegen M, Kessels RP, IntHout J, Hakobjan M, et al. BDNF polymorphism associates with decline in set shifting in Parkinson's disease. Neurobiol Aging 2015; 36: 1605.e1-6, doi: 10.1016/j.neuro biolaging.2014.08.023.

34. Mariani S, Ventriglia M, Simonelli I, Bucossi S, Siotto M, R RS. Meta-analysis study on the role of bone-derived neurotrophic factor Val66Met polymorphism in Parkinson's disease. Rejuvenation Res 2015; 18: 40-47, doi: 10.1089/ rej.2014.1612.

35. Lee YH, Song GG. BDNF $196 \mathrm{~g} / \mathrm{a}$ and $270 \mathrm{c} / \mathrm{t}$ polymorphisms and susceptibility to Parkinson's disease: a metaanalysis. J Mot Behav 2014; 46: 59-66, doi: 10.1080/ 00222895.2013.862199.

36. Zintzaras E, Hadjigeorgiou GM. The role of G196A polymorphism in the brain-derived neurotrophic factor gene in the cause of Parkinson's disease: A meta-analysis. J Human Genetics 2005; 50: 560-566, doi: 10.1007/s10038-005-0295-z.

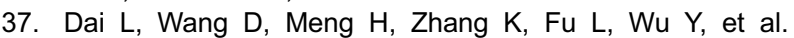
Association between the BDNF G196A and C270T polymorphisms and Parkinson's disease: a meta-analysis. Int J Neurosci 2013; 123: 675-683, doi: 10.3109/00207454. 2013.798784.

38. Emre M, Aarsland D, Brown R, Burn DJ, Duyckaerts C, Mizuno $\mathrm{Y}$, et al. Clinical diagnostic criteria for dementia associated with Parkinson's disease. Mov Disord 2007; 22: 1689-1707; quiz 1837, doi: 10.1002/mds.21507.

39. Litvan I, Goldman JG, Troster AI, Schmand BA, Weintraub $D$, Petersen RC, et al. Diagnostic criteria for mild cognitive impairment in Parkinson's disease: movement disorder society task force guidelines. Mov Disord 2012; 27: 349-356, doi: 10.1002/mds.24893. 